\title{
Avaliação da Cobertura Vacinal em crianças de 2 meses a 5 anos na Estratégia Saúde da Família
}

\section{Evaluation of Vaccination's Coverage among children aged from 2 months to 5 years in Family Health Strategy}

\author{
Samara Guerra Carneiro ${ }^{1}$ \\ Tiago Turci Ribeiro ${ }^{1}$ \\ Márcia Dorcelina Trindade Cardoso ${ }^{2}$ \\ João Francesco Strapasson ${ }^{1}$ \\ Ana Flávia Baldoni Costa ${ }^{1}$ \\ Flávia Drummond Guina ${ }^{1}$
}

\section{Artigo \\ Original}

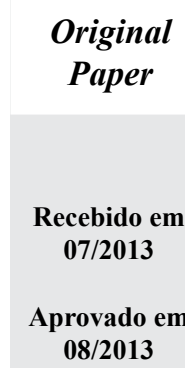

\section{Palavras-chave:}

Cobertura Vacinal

Fatores de Risco

Atenção primária à saúde

\section{Resumo:}

A vacinação é uma ação rotineira dos serviços de saúde e tem como objetivo erradicar doenças imunoprevníveis. O objetivo deste estudo foi avaliar a cobertura vacinal em crianças de 2 meses a 5 anos em quatro Unidades da Saúde da Família em Volta Redonda - RJ. Estudo transversal e descritivo, utilizando o método de amostragem por conveniência com os lactentes e pré-escolares que compareceram às unidades de saúde no período de 30 de julho a 30 de agosto de 2012. O critério de inclusão foi a idade entre dois meses a cinco anos, e o de exclusão foi a ausência do Cartão de Vacinação no momento da entrevista. Para cada criança avaliada, foi aplicado um questionário à mãe. $11 \%$ das crianças estavam em atraso com a vacinação. Sobre a idade materna, constatou-se que a maioria das mães das crianças com cartões atrasados encontravam-se na faixa etária entre 26 e 30 anos. Quanto à escolaridade materna, $64 \%$ das mães das crianças com cartões atrasados estudaram entre 4 e 7 anos. Em relação à renda familiar, 91\% das mães de crianças com cartões atrasados informaram renda entre 1 a 2 salários. Observando-se a faixa etária das crianças com os cartões atrasados, notou-se que o maior número de atrasos ocorreu em menores de um ano. Para obtermos maior êxito na cobertura vacinal, cabe aos profissionais de saúde participar mais ativamente na busca de crianças em falta com a vacinação, por meio de revisão sistemática dos cartões, de palestras ministradas à população e de uma maior efetividade nas visitas domiciliares. 
Abstract:

Vaccination is a routine action on health services and aims to eradicate preventable diseases. The aim of this study was to assess vaccination coverage in children aged from 2 months to 5years in four Family Health Care Units Volta Redonda-RJ/Brazil. Transversal and descriptive study, using convenience sampling method, with infants and preschoolers who attended at health facilities in the period from 30 July to 30 August 2012.

The attendance criterion was the age from two months to five years old and exclusion was the absence of Vaccination Card at the time of the interview. For each assessed child, a questionnaire was addressed to the mother. $11 \%$ of children were delayed with their vaccination. On maternal age, it was found that most mothers of children with delay on vaccination, were aged between 26 and 30 years. Regarding their level of schooling, 64\% of mothers of children with late booklet, went to school for 4 to 7 years.

Regarding family income, 91\% of mothers of children with delay in vaccination reported income between 1 and 2 minimum wages. Observing the age of the children with late booklet, it was noted that the greatest number of delays occurred with children of less than one year old. To obtain greater success in immunization coverage, the health professionals have to participate more actively in the search for children with missing vaccination, through a systematic review of the booklets, lectures for the population and a better effectiveness in the home visits.

\section{Introdução}

A vacinação é uma ação integrada e rotineira dos serviços de saúde, que tem como objetivo de erradicar, eliminar e controlar as doenças imunopreveníveis no território brasileiro. ${ }^{1}$ Vacinar implica em um fator de proteção específico contra doenças graves, causadoras de danos irreversíveis ou letais. Além disso, a vacinação melhora o nível de saúde de uma comunidade e isso se reflete nos indicadores, especialmente na taxa de mortalidade infantil. $^{2}$

Pertence ao nível de atenção primária de baixa complexidade, representando um dos grandes avanços da tecnologia médica nas últimas décadas, constituindo-se no procedimento de melhor relação custo e efetividade no setor saúde. ${ }^{3} \mathrm{O}$ reconhecimento da cobertura vacinal e dos fatores que levam ao atraso ou falta de imunização é essencial para a adequação de programas governamentais de vacinação e para a identificação de crianças em atraso. ${ }^{4}$

No Brasil, o Programa Nacional de Imunizações do Ministério da Saúde (PNI/
Keywords:

Immunization

Coverage

Risk factors

Primary Health Care
MS), formulado em 1973, objetivou o controle ou erradicação de doenças infectocontagiosas e imunopreviníveis, incluindo a poliomielite, o sarampo, a difteria, o tétano, a coqueluche e a tuberculose, gerando grande impacto nas condições gerais de saúde. O declínio acelerado de morbimortalidades por doenças imunopreveníveis nas décadas recentes no Brasil, em escala mundial, serve de prova incontestável do enorme benefício que é oferecido às populações por intermédio das vacinas. ${ }^{3,5}$

$\mathrm{O}$ calendário vacinal definido pelo $\mathrm{PNI} /$ MS corresponde ao conjunto de vacinas consideradas de interesse prioritário à saúde pública do país. Atualmente, é composto por 13 produtos recomendados à população, desde o nascimento até a terceira idade. São considerados quatro calendários: da criança, do idoso, do adolescente e adulto e dos povos indígenas. ${ }^{6}$ Em 2005, iniciou-se a distribuição do Cartão da Criança por maternidades públicas e privadas, em substituição à Caderneta de Vacinação aprovada em 1977.,7,

Houve uma sensível melhora na cobertura vacinal nos últimos anos, com a introdução, por exemplo, da vacina contra Rotavírus no 
Calendário Básico de Vacinação da Criança em 2006. Além disso, a partir de 2010, crianças menores de dois anos passaram a contar com a aplicação da pneumocócica 10 e meningocócica C. Por último, em agosto de 2012, houve a substituição da Vacina Oral contra Poliomielite (produzida com vírus atenuados) para a Vacina Injetável contra Poliomielite (produzida com vírus inativados). Com a utilização de vírus inativados, a possibilidade de desenvolver a doença a partir da vacina é nula, eliminando a probabilidade de a doença se desenvolver como possível a partir da vacina feita com vírus atenuados. Apesar de o risco teórico estimulado ser considerado baixo, a OMS reconhece os danos associados ao uso da vacina oral. Outra vacina que foi incluída no Calendário Básico de Vacinação da Criança foi a Pentavalente. É a atual Tetravalente que incorporou a vacina contra Hepatite B na mesma dose, diminuindo, assim, uma injeção para a criança. ${ }^{8}$

Apesar da melhoria da cobertura vacinal no Brasil nos últimos anos, existem crianças que ainda não são vacinadas corretamente mesmo em regiões em que há boa disponibilidade dos serviços de saúde. ${ }^{9}$ Assim, o Ministério da Saúde lançou a campanha de atualização das Cadernetas de Vacinação de crianças menores de 5 anos, que ocorreu no dia 18 de agosto de 2012, com o intuito de reduzir a taxa de abandono do esquema vacinal básico e, consequentemente, aumentar a cobertura vacinal no território brasileiro. ${ }^{10,11}$

Vários estudos vêm sendo realizados no Brasil e no mundo com o objetivo de elucidar essa questão da cobertura vacinal e dos fatores que levam a não vacinação. Dentre os fatores de risco, destacam-se: baixa renda familiar ${ }^{12}$, extremos de idade materna ${ }^{13}$, maior número de filhos ${ }^{13}$, baixa escolaridade materna $14,15,16,17,18$ e presença de doença na criança ${ }^{17}$, entre outros. Não apenas fatores relacionados aos usuários são considerados para a cobertura vacinal. Fatores estruturais relacionados aos serviços de saúde também podem ser levados em conta como, por exemplo, a dificuldade de agendamento e tempo de espera prolongado. ${ }^{16}$

Esse estudo teve como objetivo avaliar a cobertura vacinal em crianças de 2 meses a 5 anos em quatro UBSF na cidade de Volta Redonda - RJ e analisar os potenciais fatores contribuintes para o atraso vacinal.

\section{Materiais e Métodos}

O presente trabalho foi realizado em quatro unidades básicas de saúde de família (UBSF) do município de Volta Redonda, RJ.

A UBSF do bairro Volta Grande é composta por cinco equipes, e a UBSF Santo Agostinho é composta por três, enquanto que as UBSF dos bairros Vila Americana e Nova Primavera possuem apenas uma equipe. Cada equipe possui um médico, um enfermeiro, um técnico de enfermagem e de quatro a seis agentes comunitários de saúde.

Foi realizado estudo transversal e descritivo, utilizando o método de amostragem por conveniência com as crianças de 2 meses a 5 anos que compareceram às unidades de saúde no período de 30 de julho a 30 de agosto de 2012 para consultas médicas ou para vacinação. $O$ critério de inclusão foi a idade entre 2 meses a 5 anos, e as crianças que não portavam o Cartão de Vacinação no momento da entrevista foram excluídas.

Para cada criança avaliada, foi aplicado um questionário autoexplicativo e padronizado (anexo 1) à mãe ou responsável.

Para a avaliação do cumprimento do esquema de vacinação, foram utilizadas as definições preconizadas pelo Ministério da Saúde para o calendário básico de vacinação da criança aos 5 anos, disposto na Tabela $1 .^{6}$ Apesar do Calendário Básico de Vacinação ter sido alterado no dia dezoito de agosto de 2012, esse não foi avaliado neste trabalho, pois consideramos em atraso as crianças que estavam em falta com a vacinação há um mês ou mais. 


\begin{tabular}{|c|c|c|c|c|c|c|c|c|c|c|}
\hline VACINAS & & & & IDADH & E NÚN & [ERO D & E DOS & & & \\
\hline & Ao nascer & $1^{\circ}$ mês & $2^{\circ}$ mês & $3^{\circ}$ mês & $4^{\circ}$ mês & $5^{\circ}$ mês & $6^{\circ}$ mês & $12^{\circ}$ mês & $15^{\circ}$ mês & 4 anos \\
\hline BCG & DU & & & & & & & & & \\
\hline Hepatite B & $1^{\mathrm{a}}$ dose & $2^{\circ}$ dose & & & & & $3^{\circ}$ dose & & & \\
\hline DTP & & & $1^{\mathrm{a}}$ dose & & $2^{\circ}$ dose & & $3^{\circ}$ dose & & Reforço & $2^{\circ}$ Reforço \\
\hline $\mathrm{Hib}$ & & & $1^{\mathrm{a}}$ dose & & $2^{\circ}$ dose & & $3^{\circ}$ dose & & & \\
\hline VOP (polio) & & & $1^{\mathrm{a}}$ dose & & $2^{\circ}$ dose & & $3^{\circ}$ dose & & Reforço & \\
\hline VORH (rotavírus) & & & $1^{\mathrm{a}}$ dose & & $2^{\circ}$ dose & & & & & \\
\hline Pneumocócica 10 & & & $1^{\mathrm{a}}$ dose & & $2^{\circ}$ dose & & $3^{\circ}$ dose & Reforço & & \\
\hline Meningocócica C & & & & $1^{\mathrm{a}}$ dose & & $2^{\circ}$ dose & & & Reforço & \\
\hline SCR(tríplice viral) & & & & & & & & $1^{\mathrm{a}}$ dose & & $2^{\circ}$ dose \\
\hline
\end{tabular}

Nota: Fonte: Ministério da Saúde (Brasil). Portal da saúde SUS. Calendário de vacinação atende a todas as idades. [Citado em: 13 agosto 2012]. Disponível em: http://portalsaude.saude.gov.br/portalsaude/ noticia/3591/162/calendario-de-vacinacao-atende-a-todas-as-idades.html.

As doses de vacinas foram classificadas como confirmadas quando comprovadas no Cartão da Criança pelo carimbo do posto de vacinação, data e a rubrica do funcionário.

\section{Resultados}

Foram avaliadas 96 Cadernetas de Vacinação nos quatro postos de saúde, sendo 30 do bairro Volta Grande, 21 do bairro Vila Americana, 23 cartões do bairro Santo Agostinho e 22 do bairro Nova Primavera. Do total (96), 11 crianças estavam em atraso, configurando $11 \%$, enquanto 85 encontravam-se em dia, representando 89\%. A UBSF Santo Agostinho foi a que apresentou maior atraso no esquema vacinal, apontando $22 \%$ (5) das 23 crianças avaliadas. A UBSF Nova Primavera mostrou um atraso compatível com 18\% (4) do total de 22 e a UBSF Vila Americana apresentou 10\% (2) de atraso da totalidade de 21 cartões. Já a UBSF Volta Grande não revelou atraso vacinal nas 30 crianças analisadas, configurando $100 \%$ de cartões em dia.

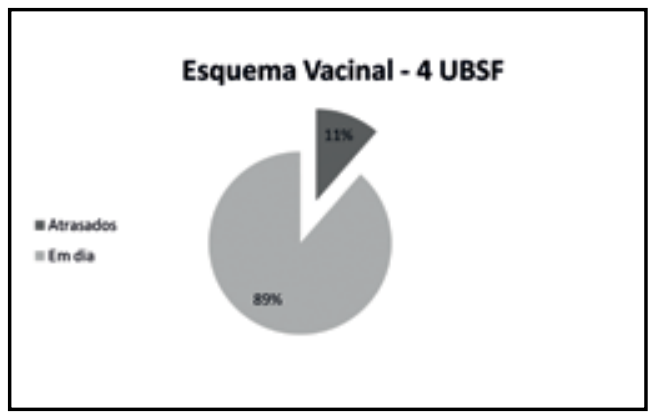

Figura 1: Esquema vacinal nas 4 UBSF.

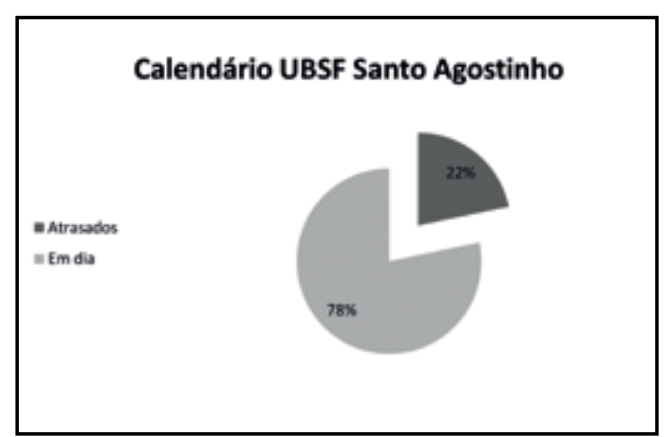

Figura 2: Calendário Vacinal na UBSF Santo Agostinho.

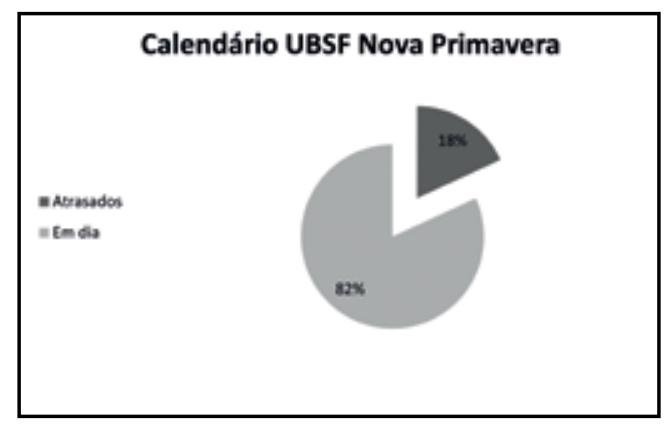

Figura 3: Calendário Vacinal na USF Nova Primavera.

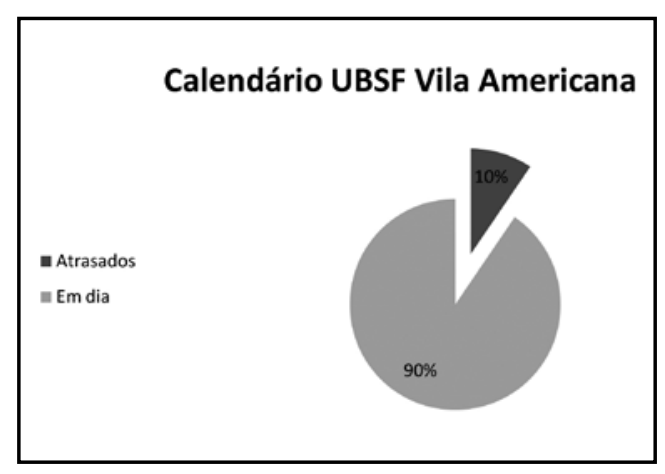

Figura 4: Calendário Vacinal na UBSF Vila Americana. 


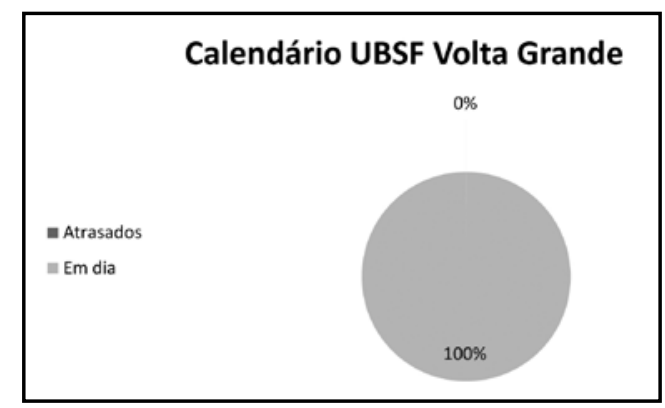

Figura 5: Calendário Vacinal na UBSF Volta Grande.

Dos cartões atrasados (11), 45\% (5) pertenciam a crianças do sexo feminino e $55 \%$ (6) do masculino.

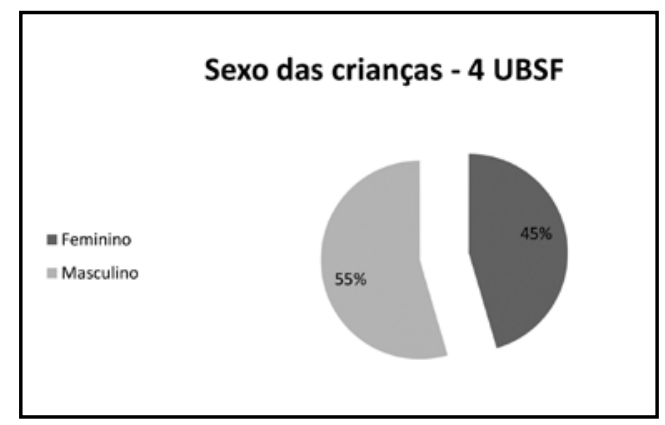

Figura 6: Sexo da crianças em atraso nas 4 UBSF.

Em relação à idade das crianças que estavam em atraso vacinal, a faixa etária de maior prevalência foi a de 6 meses a 1 ano, correspondendo a $55 \%$, seguida de $27 \%$ de crianças de 1 a 2 anos e $9 \%$ está na faixa etária de 0 a 6 meses e também de 2 a 3 anos.

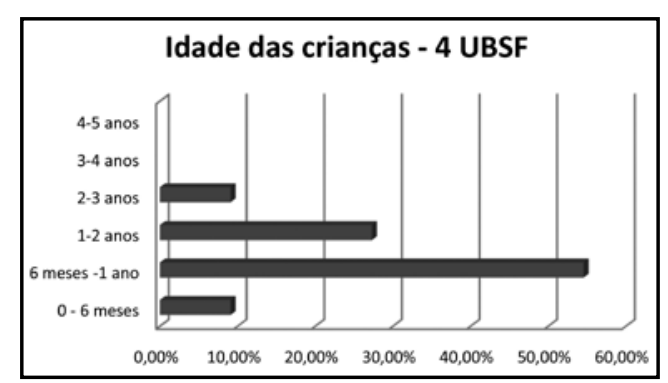

Figura 7: Idade das crianças em atraso na 4 UBSF.

Considerando crianças com cartões atrasados, a faixa etária materna predominante nas 4 UBSF analisadas foi a de 26 a 30 anos (46\%), seguida de 36\% de mães entre 21-25 anos e $18 \%$ entre $16-20$ anos.

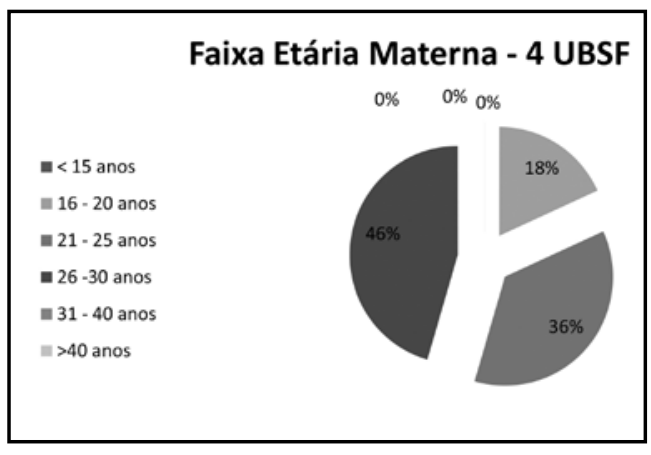

Figura 8: Faixa Etária Materna nas 4 UBSF.

Quanto à escolaridade, avaliada em anos de estudo, a maioria (64\%) das mães das crianças com cartões atrasados estudaram entre $4 \mathrm{e}$ 7 anos, e $36 \%$ estudaram de 8 a 11 anos.

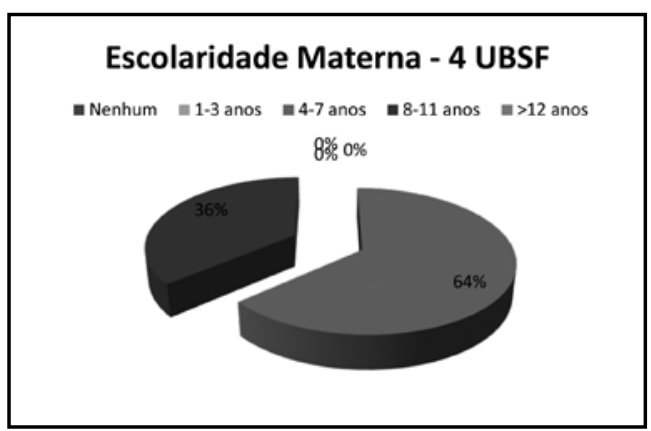

Figura 9: Escolaridade Materna nas 4 UBSF.

Nas 4 UBSF avaliadas, a renda familiar em número de salários mínimos - das famílias das crianças com cartão de vacinação atrasado foi de $91 \%$ entre 1 e 2 salários e $9 \%$ entre 3 e 4 salários.

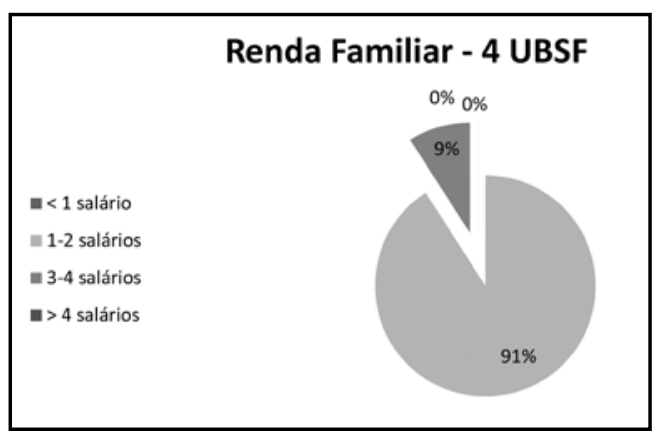

Figura 10: Renda Familiar nas 4 UBSF.

Levando em consideração o número de irmãos das crianças em atraso nas 4 UBSF, a maioria (64\%) possuía 2 irmãos, seguida de $27 \%$ que não tinham irmãos e $9 \%$ com apenas 1 . 


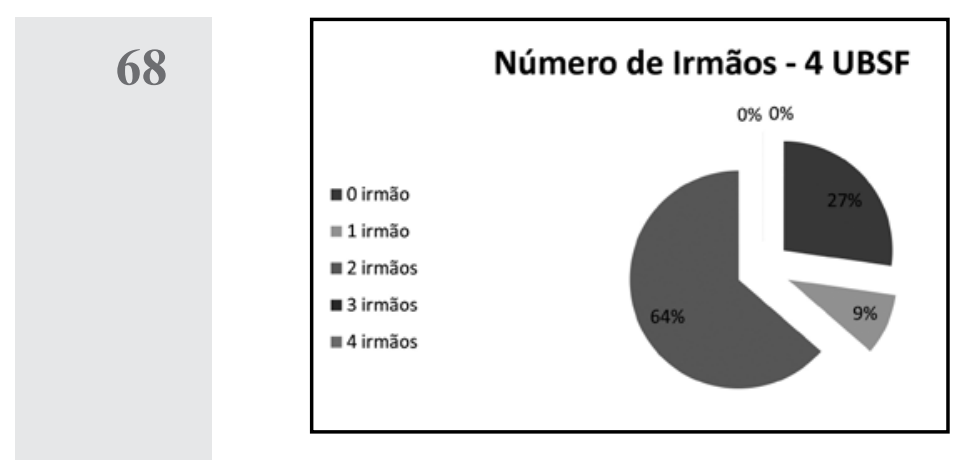

Figura 11: Número de irmãos das crianças em atraso nas 4 UBSF.

Quando questionadas sobre o motivo da falha vacinal, $37 \%$ das mães de crianças em atraso nas 4 UBSF indicaram o esquecimento como principal motivo. $27 \%$ classificaram a dificuldade de acesso ao posto e $18 \%$ das mães alegaram que a criança estava doente na data prevista. O 18\% restantes alegaram outros motivos.

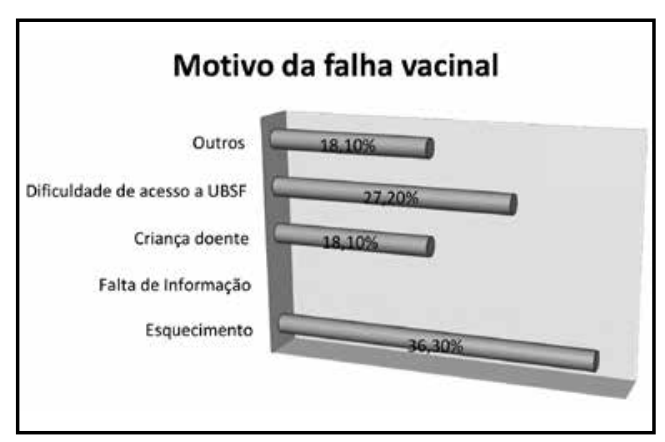

Figura 12: Motivo da falha vacinal.

Analisando os Cartões de Vacinação, constatou-se atraso nas seguintes vacinas (Tabela 2):
TABELA 2: DOSES DAS VACINAS ATRASADAS.

\begin{tabular}{lll}
\hline Vacina & Dose & $\begin{array}{l}\text { Número de } \\
\text { atrasos }\end{array}$ \\
\hline Hepatite B & $3^{\text {a dose }}$ & 1 \\
Rotavírus & $1^{\text {a dose }}$ & 2 \\
Rotavírus & $2^{\text {a dose }}$ & 3 \\
-VOP & $2^{\text {a dose }}$ & 1 \\
-VOP & Reforço & 1 \\
Tetravalente & $2^{\text {a dose }}$ & 2 \\
Tetravalente & $3^{\text {a dose }}$ & 1 \\
DTP & $1^{\circ}$ reforço & 2 \\
-Pneumocócica 10 & $2^{\text {a dose }}$ & 4 \\
-Pneumocócica 10 & $3^{\text {a dose }}$ & 2 \\
-Pneumocócica 10 & Reforço & 2 \\
Meningo C & $1^{\text {a dose }}$ & 1 \\
Meningo C & $2^{\text {a dose }}$ & 2 \\
Meningo C & Reforço & 1 \\
Tríplice Viral & $1^{\text {a dose }}$ & 1
\end{tabular}

\section{Discussão}

Observamos que $11 \%$ de todas as crianças analisadas não estavam em dia com a vacinação. Ao avaliarmos separadamente cada UBSF, percebemos que a de maior atraso foi a de Santo Agostinho, seguida das UBSF de Nova Primavera e Vila Americana, respectivamente, enquanto a UBSF Volta Grande não apresentou Carteira de Vacinação em atraso.

Diante de tal situação, podemos relatar que a UBSF de maior atraso está em período de reforma, em local provisório e sem a adequada instalação, podendo esses ser considerados possíveis fatores para o maior atraso vacinal, já que a maioria das mães de crianças em atraso relatou dificuldade de acesso ao posto como motivo da falha vacinal. Como foi observado por Rahman et al. (1995), fatores estruturais relacionados aos serviços de saúde também podem ser considerados como agravantes do atraso vacinal. ${ }^{16}$

Já as UBSF de Vila Americana e Nova Primavera são compostas por apenas 1 equipe de saúde, fator que pode ter contribuído com o atraso, uma vez que as equipes podem não estar sendo eficientes na integração com a população. Contudo, a UBSF Volta Grande, que apresenta melhor infraestrutura, boa localização e maior número de equipes, teve o melhor resultado. 
Considerando a faixa etária de crianças com carteiras de vacinação em atraso, analisamos que a de maior prevalência foi a de 6 meses a 1 ano, valendo ressaltar a importância da vacinação nessa idade, já que é uma faixa etária bastante suscetível a infecções e, uma vez instalada a doença, a criança pode sofrer a incidência de danos irreversíveis ou letais. ${ }^{2}$

Sobre a idade materna, segundo Tertuliano e Stein (2011), o maior percentual de atrasos ocorreu nos extremos de idade materna. ${ }^{13}$ No presente estudo, constatou-se que a faixa etária materna dominante foi a de 26 a 30 anos, contradizendo os autores citados.

A respeito da escolaridade materna, observamos que o maior percentil de atraso foi observado nos cartões de crianças cujas mães têm apenas de 4 a 7 anos de estudo, o que pode ser confirmado por outros estudos. ${ }^{14,15,16,17,18}$ Para Silva et al. (1999), a baixa escolaridade materna é fator associado a maiores coeficientes de morbimortalidade infantil e pior desenvolvimento físico das crianças, além de contribuir com maior número de faltas em consultas preventivas de puericultura. Com o aumento da escolaridade materna, há a tendência de diminuição de crenças em superstições e explicações mágicas para as doenças, assim, mães com mais de 8 anos de escolaridade adaptam-se melhor à tecnologia médica e tendem a fazer uso mais efetivo dos serviços de saúde. ${ }^{9}$

A cobertura vacinal mostrou-se mais prejudicada em segmentos economicamente menos privilegiados da população, já que $91 \%$ das crianças em atraso pertenciam a famílias cuja renda era entre 1 e 2 salários mínimos. Além disso, percebemos que o maior número de irmãos da criança também foi um fator que contribui para a falha. Segundo Tertuliano e Stein (2011), alguns fatores como os citados podem levar as mães a não vacinarem adequadamente seus filhos, uma vez que essas enfrentam numerosas dificuldades diariamente tendo que cuidar dos trabalhos domésticos. Sua atenção muitas vezes fica centralizada na garantia da alimentação e na busca de tratamento para os problemas de saúde. Em contrapartida, as atividades preventivas não são tratadas como prioridade. ${ }^{13}$

Considerando a Tabela 2, podemos observar que a maioria das doses de vacinas atrasadas se deu com as vacinas Pneumocócica 10 e contra Rotavírus. De acordo com um estudo realizado por Tertuliano e Stein (2011), as vacinas que apresentavam três doses ou mais para a imunização das crianças apresentam um percentual de atraso mais elevado, que justifica o atraso da vacina pneumocócica. ${ }^{13} \mathrm{~A}$ observação de atraso na aplicação das vacinas, especialmente das segundas doses e reforço, aponta a necessidade de melhoria das estratégias de busca de faltosos.

\section{Conclusão}

Após o término do presente estudo, podemos notar que a cobertura vacinal nos postos de saúde analisados ainda não é efetiva. Percebemos que o atraso vacinal de $11 \%$ nas UBSF avaliadas realmente se relaciona com os fatores de risco considerados, tais como, baixa renda familiar ${ }^{12}$, maior número de filhos ${ }^{13}$, baixa escolaridade materna ${ }^{14,15,16,17,18} \mathrm{e}$ presença de doença na criança ${ }^{19}$, entre outros. No entanto, nota-se a exceção do fator de risco relacionado à idade materna, uma vez que, em nossa análise, o maior percentual de atraso foi observado na faixa etária entre 26 e 30 anos, contrariando outros estudos citados. Além disso, notamos que a menor estrutura da UBSF e menor equipe de saúde, também contribuem para a falha do esquema vacinal.

$\mathrm{O}$ ato de vacinar implica em uma interação entre os responsáveis pela criança e os serviços de saúde. O Ministério da Saúde sugere algumas ações que devem ser seguidas para acabar com as perdas de oportunidades em vacinação. ${ }^{9}$

Sendo assim, para obtermos maior êxito na cobertura vacinal, cabe aos profissionais de saúde participar mais ativamente na busca de crianças em falta com a vacinação, através da revisão sistemática dos cartões, de uma maior efetividade nas visitas domiciliares, cabendo um maior comprometimento aos agentes de saúde. Outra estratégia para o aumento da cobertura vacinal é a melhoria das atividades de educação em saúde, com o intuito de difundir informações aos grupos alvo, uma vez que a falta de conhecimento e crenças da população contribuem para o atraso vacinal. 9,13 


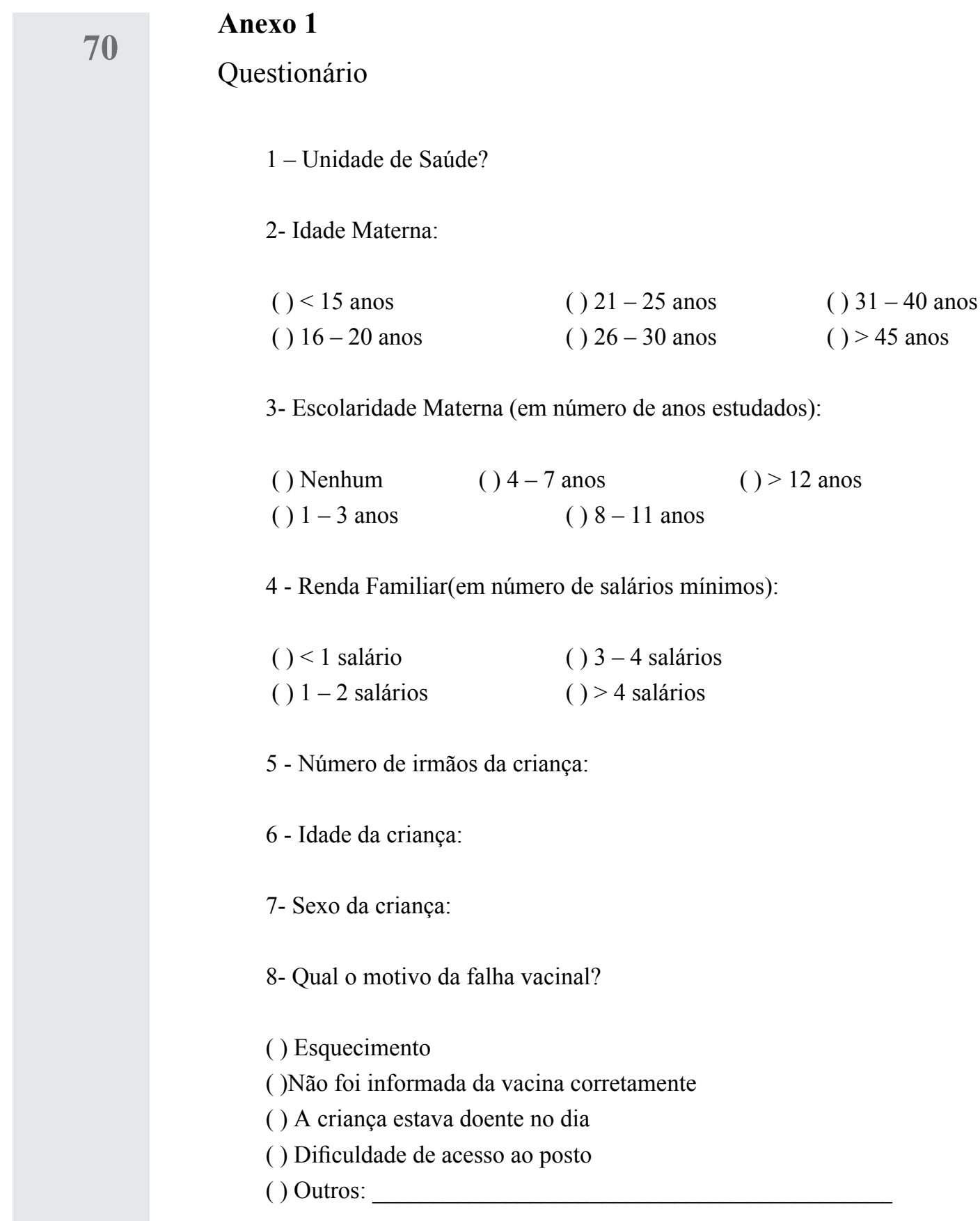




\section{Referências}

1. MINISTÉRIO DA SAÚDE (Brasil). Portal da saúde SUS. Vacinação. Brasil: Ministério da saúde; [Citado em: 22 agosto 2012]. Disponível em: http://portal.saude. gov.br/portal/saude/visualizar_texto. cfm?idtxt=29489. Acesso em 22 ago. 2012.

2. GATTI, M. A. N.; OliVEIRA, L. R. Crianças faltosas à vacinação, condições de vida da família e concepção sobre vacina: um inquérito domiciliar. Salusvita, Bauru, v. 24, p. 427-436, 2005.

3. GUimARÃES, T. M. R.; ALVES, J. G. B.; TAVARES, M. M. F. Impacto das ações de imunização pelo Programa Saúde da Família na mortalidade infantil por doenças evitáveis em Olinda, Pernambuco, Brasil. Cad. Saúde Publica, Rio de Janeiro, v. 24, n. 4, p. 868-876, 2009.

4. HENDERSON, R. H.; KEJA, J.; HAYDEN, G.; GALAKZA, A.; CLEMENTS, J.; CHAN, C. Immunizing the children of the world: progress and prospects. Bull World Health Organ, Switzerland, v. 66, p. 535-543, 1988.

5. MINISTÉRIO DA SAÚDE (Brasil). Programa Nacional de Imunizações 30 anos. Brasília: Ministério da Saúde; 2003.

6. MINISTÉRIO DA SAÚDE (Brasil). Portal da saúde SUS. Calendário de vacinação atende a todas as idades. Disponível em: http://portalsaude.saude. gov.br/portalsaude/noticia/3591/162/ calendario-de-vacinacao-atende-a-todasas-idades.html. Acesso em 13 ago.2012.

7. MINISTÉRIO DA SAÚDE (Brasil). Dois séculos de vacina no Brasil. Brasil: Revista da Vacina. Disponível em: $<$ http://www.ccs.saude.gov.br/revolta/ ltempo.html>. Acesso em 29 ago. 2012.
8. O SUL DE MINAS (Minas Gerais). Ministério da saúde inclui novas vacinas no calendário infantil. Disponível em: http://www.osuldeminas.com/ osuldeminas/Pagina.do;jsessionid $=\mathrm{e} 7 \mathrm{on}$ cjzazcs 9 ?idSecao $=29 \&$ idNoticia $=8080$. Acesso em 12 ago. 2012.

9. SILVA, A. A. M.; UILHO, A. G.; TONIAL, S. R.; SILVA, R. A. Cobertura vacinal e fatores de risco à não-vacinação em localidade urbana do Nordeste brasileiro, 1994. Revista Sáude Pública, São Paulo, v. 33, n. 2, p. 147-156, 1998.

10. CONEXÃO TOCANTINS (Tocantins). Crianças menores de 5 anos devem atualizar caderneta de vacinas neste sábado. Disponível em: http://conexaoto. com.br/2012/08/17/criancas-menoresde-5-anos-devem-atualizar-cadernetade-vacinas-neste-sabado. Acesso em 14 ago.2012.

11. MINISTÉRIO DA SAÚDE (Brasil). Brasil: Ministro lança campanha para atualizar caderneta de vacinação. Disponível em: http://portalsaude.saude. gov.br/portalsaude/noticia/6633/162/ ministro-lanca-campanha-para-atualizarcaderneta-de-vacinacao.htm. Acesso em 13 ago.2012.

12. BECKER, R. A.; LECHTIG, A. Vacinação. In: SILVA, R. M. R.; coordenador. Perfil estatístico de crianças e mães no Brasil: situação de saúde 1981. IBGE, Rio de Janeiro, 1984.

13. TERTUlianO, G. C.; STEIN, A. T. Atraso vacinal e seus determinantes: um estudo em localidade atendida pela Estratégia Saúde da Família. Ciência \& Saúde Coletiva, Manguinhos, v. 16, n. 2, p. 523-530, 2011.

14. BEMFAM (Brasil). Pesquisa nacional sobre demografia e saúde 1996. Rio de Janeiro: Bemfam; 1997. 
15. CUTTS, F.; SOARES, A.; JECQUE, A. V.; CLIFF, J.; KORTBEEK, S.; COLOMBO, S. The use of evaluation to improve the Expanded Programme on Immunization in Mozambique. Bull World Health Organ, Switzerland, v. 68, p. 199-208, 1990.

16. RAHMAN, M. M.; ISLAM, M. A.; MAHANABIS, D. Mother's knowledge about vaccine preventable diseases and immunization coverage of a population with high rate ofilliteracy. J Trop Pediatr., v. 41, p. 376-81, 1995.

17. SZWARCWALD, C. L.; VALENTE, J. G. Avaliação da cobertura de vacinação em Teresina - Piauí (Brasil - 1983). Cad. Saúde Pública, Rio de Janeiro, v. 1, p. 4149, 1985.

18. LUHM, K. R.; CARDOSO, M. R. A.; WALDMAM, E. A. Cobertura vacinal em menores de dois anos a partir de registro informatizado de imunização em Curitiba, PR. Rev. Saúde Pública, São Paulo, v. 45, n. 1, 2011. 